\title{
On MP-Rings
}

Raida D. Mahmood

raida.1961@uomosul.edu.iq
Azhar M. Hajo

azhar.mohammed911@gmail.com

Department of Mathematics

College of Computer Science and Mathematics

University of Mosul, Mosul, Iraq

Received on: $28 / 11 / 2018$

Accepted on: 23/06/2019

\section{ABSTRACT}

An ideal $\mathrm{I}$ of a ring $\mathrm{R}$ is said to be right (left) Pure if for every $\in I$, there is $b \in I$ such that $a=a b$ ( $a=b a$ res). A ring $\mathrm{R}$ is said to be right (left) MP-ring, if every maximal right (left) ideal of $\mathrm{R}$ is a left (right) pure. In this paper have been studied some new properties of MP-rings, there connections with strongly regular rings.

Some of the main result of the present work are as follows:

1- Let $\mathrm{R}$ be aright MP-ring, $\mathrm{r}(\mathrm{a})$ is a W-ideal for all $a \in R$ then

$a$ - Every essential ideal is a direct summand.

$b$ - $\mathrm{R}$ is strongly regular ring.

2- Let $\mathrm{R}$ be aright MP-ring. If $\mathrm{R}$ is right almost abelian left NBF ring, then $\mathrm{R}$ is strongly regular.

Keywords: MP-ring, strongly regular, W-ideal, NBF-ring.

$$
\text { حول الحلقات من النمط-MP }
$$

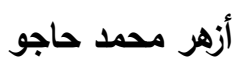

رائدة داؤد محمود

$$
\text { كلية علوم الحاسوب والرياضيات الريات الموصل، الموصل، العراق }
$$

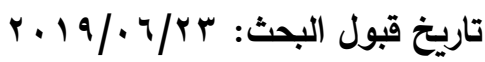

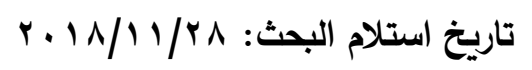

\section{الملخص}

يُقال لِلمثالي في الحلقة R ، بأَنـه نقي أَيمن (أَيسر)، إِذا كانَ لِكل a

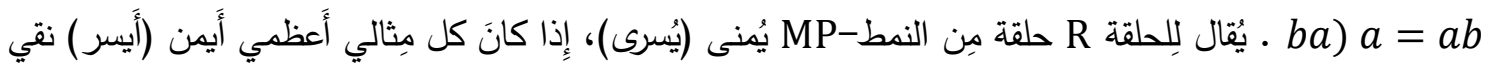
أَيَسر (أَيمن). في هذا البحث درس بعض الخواص الجديدة لِهذه الحلقات وعلاقتها مع الحلقات المنتظمة بِقوة. ومِن أَبرز النتائج التي حَصلنا عليها:

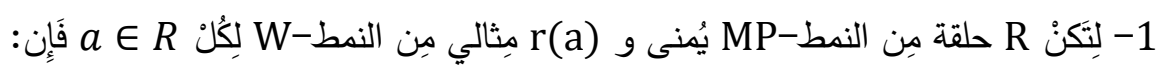

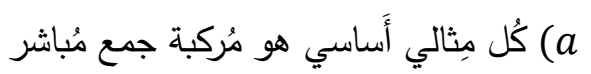
R ( $\mathrm{R}$

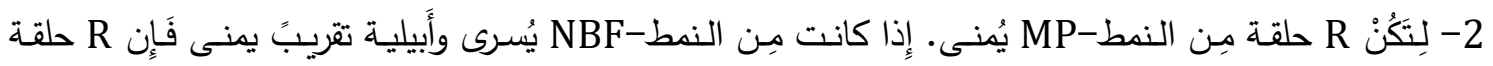
مُنتظمة بِقوة. الكلمات المفتاحية: حلقة من النمط-MP، منتظمة بقوة، مثالي من النمط-W، حلقة من النمط-NBF 
لتكن R حلقة تحوي على العنصر المحايد، والمقاسات كلها مقاسات يمنى أحادية. يُقال لِلمثالي I في الحلقة

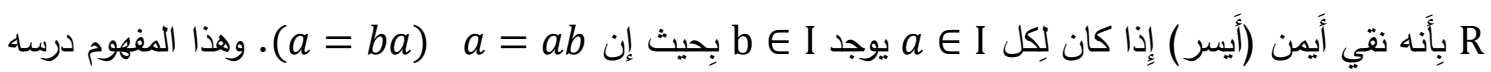

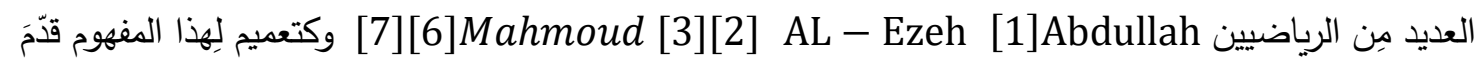
[5]Mahmood مَفهوم الحلقات مِن النمط-MP ؛ إذ يُعال لِلحلقة RP بِأَنها مِن النمط-MP يُمنى إِذا كان كُل

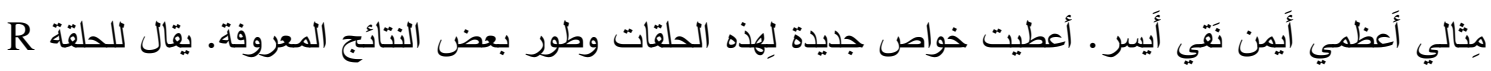

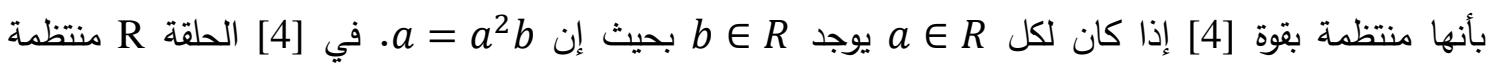

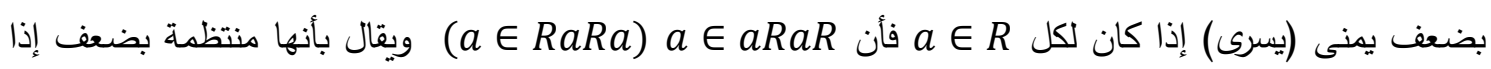
كانت R منتظمة بضعف يمنى و يسرى ـ الحلقة Rختزلة اذا كانت لا تحتوي على عناصر غير صفرية معدومة

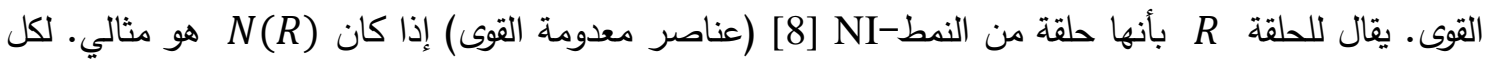

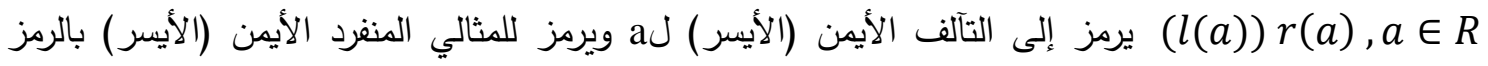
. $(Z(R)) Y(R)$

\section{2. الحلقات من النمط-MP}

ندرس في هذا البند الحلقات مِن النمط-MP وبعضاً مِن خواصها الأساسية وعلاقتها مع الحلقات المُنتظمة

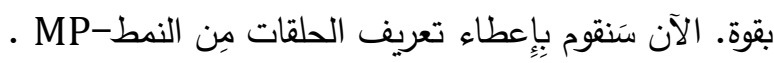

[5] [2.1) : تعريف

يُقال لِلحلقة R بِأَنها حلقة من النمط-MP يُمنى (يُسرى)، إِذا كان كل مِثالي أَعظمي أَيمن (أَيسر) نقياً أَيسر (أَيمن). مثال:- لِنكن $Z_{2}$ حلقة الأَعداد الصحيحة معيار 2 ولتكن

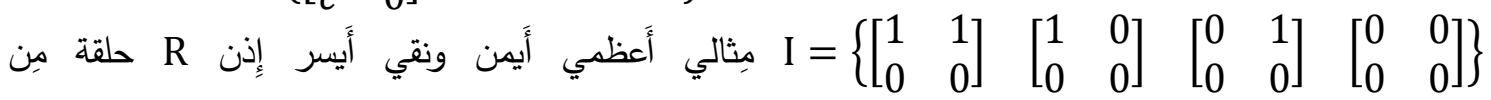
النمط-MP يمنى.

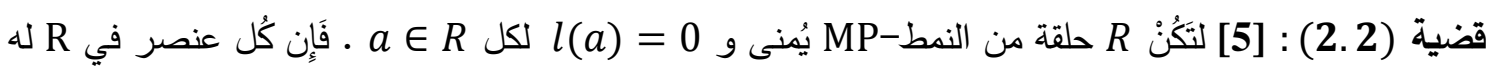
معكوس أَيمن. كُل حلقة مُنتظمة بِقوة تكون حلقة مِن النمط-MP لكن العكس غير صحيح. في المصدر [5] أعطيت المُبَرهنة الآتية:

صبرهنة (2.3) : الحلقة R مُنتظمة بِقوة إِذا وفقط إذا كانت R مُختزلة ومِن النمط-MP يُمنى تعريف: (10) : [10)

يُقال لِلمثالي الأَيمن (الأَيسر) K لكِل 
مثال: لتكن $R=\left\{\left[\begin{array}{cccc}a & a_{1} & a_{2} & a_{3} \\ 0 & a & a_{4} & a_{5} \\ 0 & 0 & a & a_{6} \\ 0 & 0 & 0 & a\end{array}\right]: a, a_{i} \in Z_{2}, i=1,2,3,4,5,6\right\}$ حلقة بعنصر محايد . لتكن $\left[\begin{array}{llll}0 & 1 & 0 & 0 \\ 0 & 0 & 1 & 0 \\ 0 & 0 & 0 & 0 \\ 0 & 0 & 0 & 0\end{array}\right] \in L,\left[\begin{array}{cccc}0 & 0 & 0 & 0 \\ 0 & 0 & 0 & 0 \\ 0 & 0 & 0 & 1 \\ 0 & 0 & 0 & 0\end{array}\right] \in L=\left\{\left[\begin{array}{cccc}0 & b_{1} & b_{2} & b_{3} \\ 0 & 0 & b_{1} & 0 \\ 0 & 0 & 0 & 0 \\ 0 & 0 & 0 & 0\end{array}\right]: b_{i} \in Z_{2}, i=1,2,3\right\}$ ومن ثََّّ Lليس مثالياً في $R$ لكنها مثالي من $\left[\begin{array}{llll}0 & 1 & 0 & 0 \\ 0 & 0 & 1 & 0 \\ 0 & 0 & 0 & 0 \\ 0 & 0 & 0 & 0\end{array}\right]\left[\begin{array}{llll}0 & 0 & 0 & 0 \\ 0 & 0 & 0 & 0 \\ 0 & 0 & 0 & 1 \\ 0 & 0 & 0 & 0\end{array}\right]=\left[\begin{array}{llll}0 & 0 & 0 & 0 \\ 0 & 0 & 0 & 1 \\ 0 & 0 & 0 & 0 \\ 0 & 0 & 0 & 0\end{array}\right] \notin L$ النمط-W

المبرهنة الآتية تُعطي أَحد الشروط لِلحلقة مِن النمط- MP يُمنى لِلحصول على الحلقة المنتظمة بِقوة.

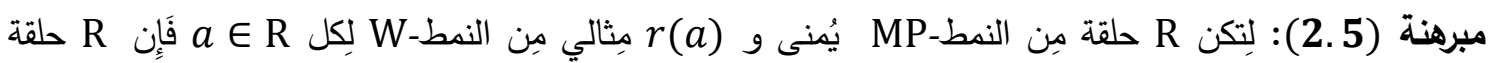
مُنتظمة بِقوة.

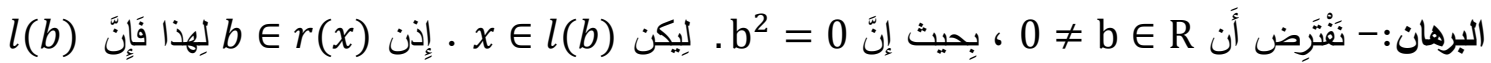

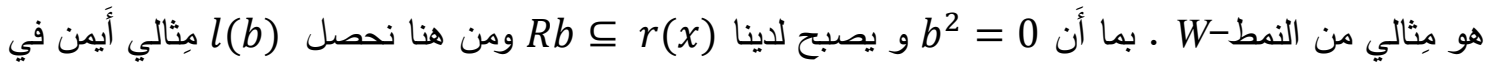

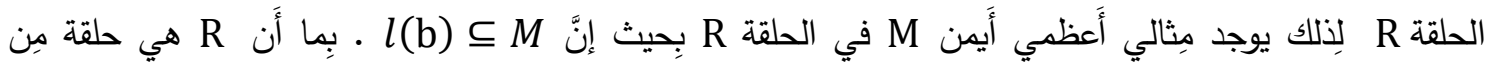

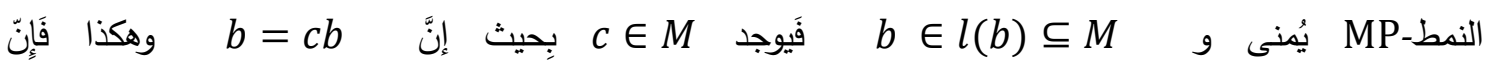

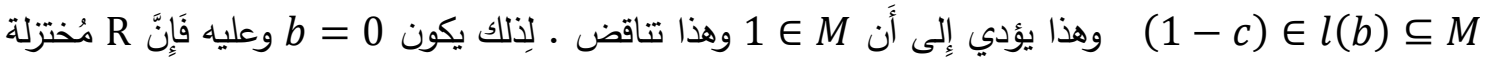

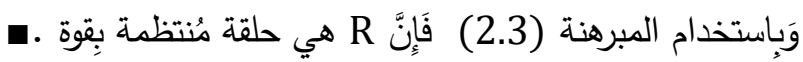

يُقال للحلقة R بِأَنها حلقة موحدة (Uniform ring) إِذا كان كُل مِثَالي غير صفري في R أَساسياً. [4]

قضية مساعدة (2.6) :[4] إذا كانت R ملقة مُختزلة فَإنَّ $\mathrm{a} \in R$ لك $r(a)=l(a)-1$

-. $a \in R$ لِّل $a R \cap r(a)=0-2$

a

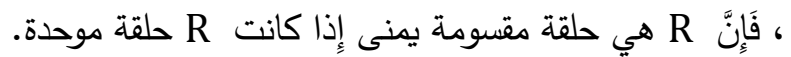

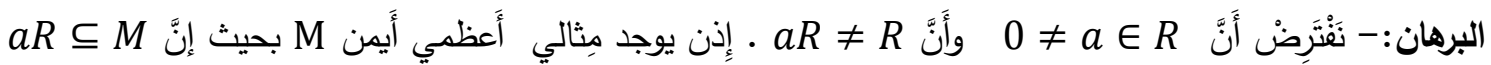

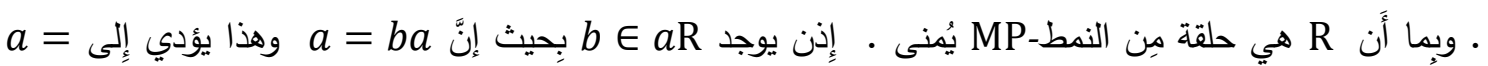

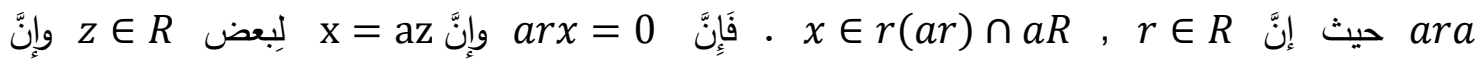

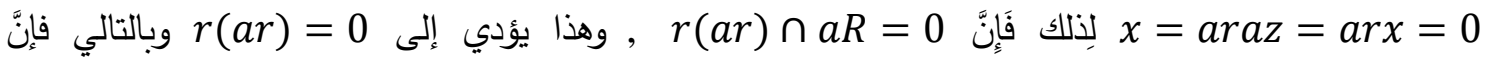
arv = 1 l(ar)=0

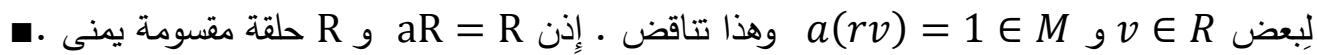

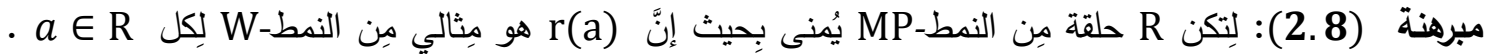

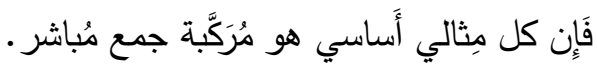


البرهان:- مِن المبرهنة (2.5) نحصل على R مُختزلة ـ لِكي نُبرهن أَن bR هو مُركبة جمع مباشر في R R م نَدعي أَن

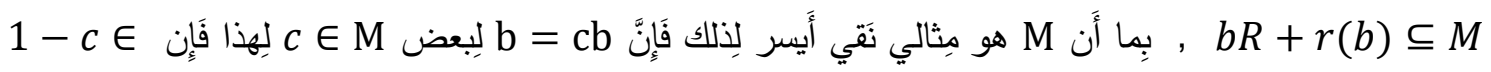

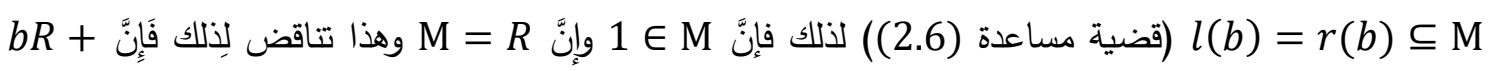

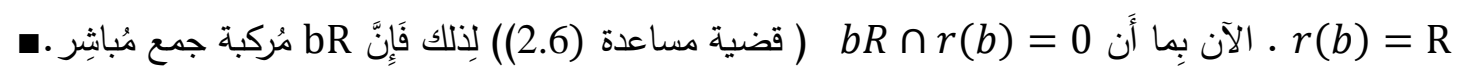

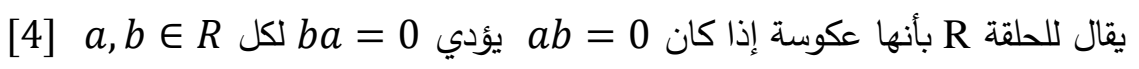

قضية (2.9): إذا كانت R حلقة عكوسة مِن النمط- MP يمنى فَإنَّ R هي مُنتظمة بِضعف يُمنى.

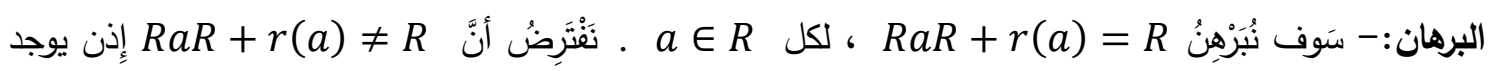

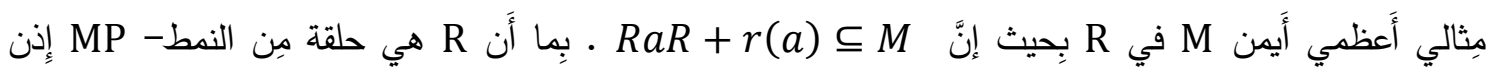

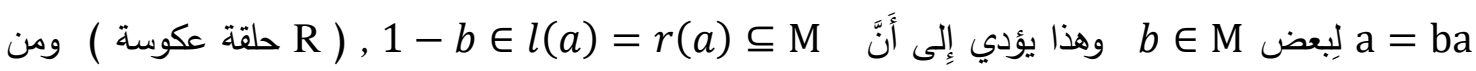

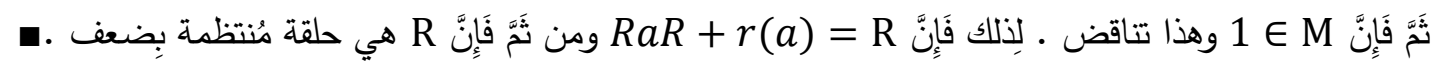

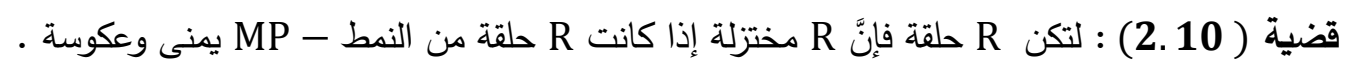
البرهان : ليكن

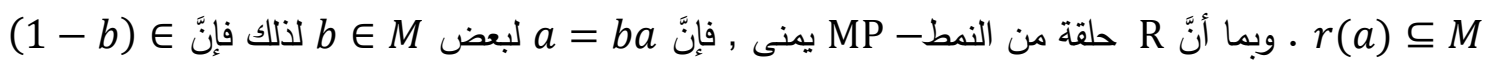

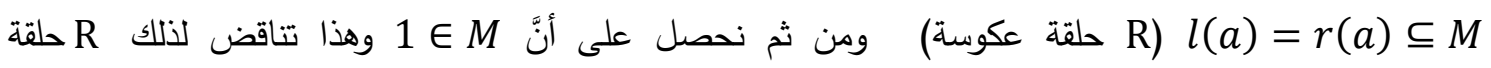

صختزلة.

تعريف (2.11): الحلقة R تُسمى أَبيلية تقريبا يُمنى (almost abelian ring) إذا كان 0 (2.1)

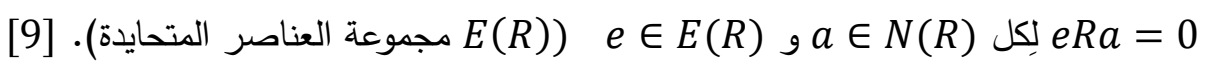
مِن الواضح أَن الحلقة أَبيلية هي أَبيلية تقريباً يُمنى .العكس غير صحيح. [10Ra]

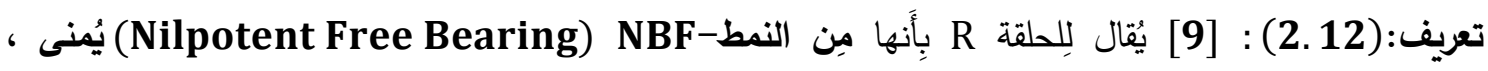
(يُعسرى) إِذا كان لِكُلِّ (R) $e b=b$

الآن نستخدم شرطاً آخر لكي تكون الحلقة- MP منتظمة بقوة.

مبرهنة: (2.13) : لِنَكُنْ R حلقة مِن النمط-MP يُمنى . إِذا كانت R مِن النمط-NBF يُسرى وأَبيلية تقريباً فَإِنها حلقة مُنتظمة بِقوة. البرهان:- نَفترض أَنَّ R غير مُختزلة . لِهذا يوجد

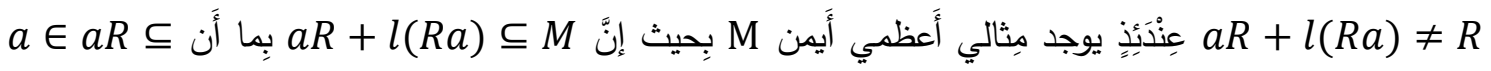

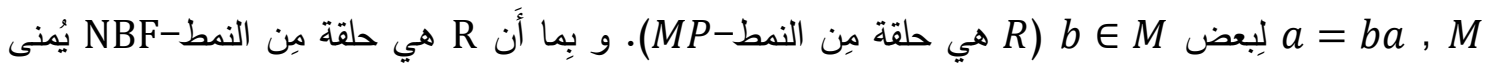

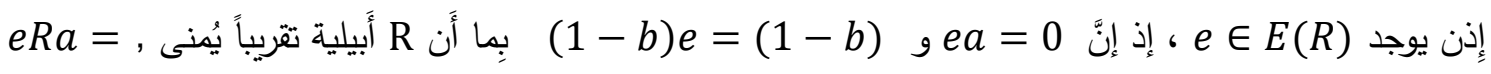

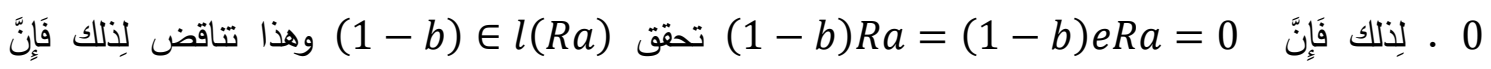

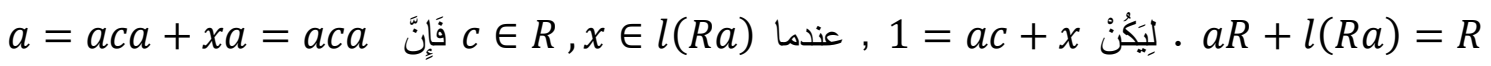

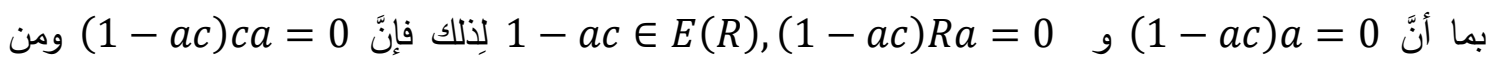


هنا نحصل على

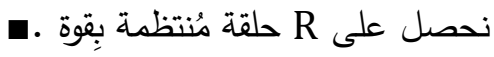

تعريف (2.14):يُقال لِلمقاس الأَيْن M (nil - injective) nil- على الحلقة R بِأَنه غامر مِن إذا كان لِكُلْ (R) حيث $f=m \in M$ ،يُقال لِلحلقة $R=M$ [8].nil-النمط قبل الإنتهاء مِن هذا البند نوجد شرط الحلقات الغامرة التي تكون من النمط-nil تقريباً يمنى لكي تكون

منتظمة بقوة.

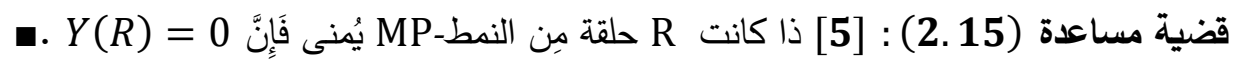

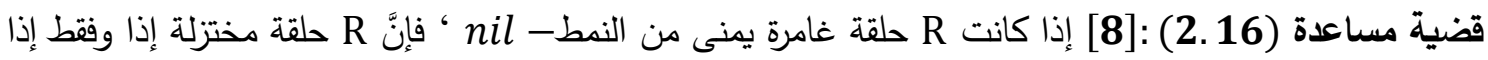
كانت من النمط مبرهنة (2.17) : ليكن N(R) مثالي في الحلقة R, فإنَّ العبارتين الآتيتين متكافأتان: R-1

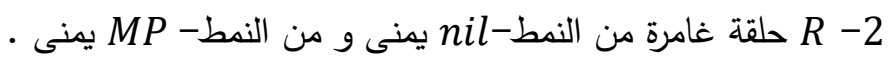

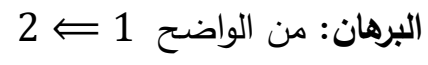

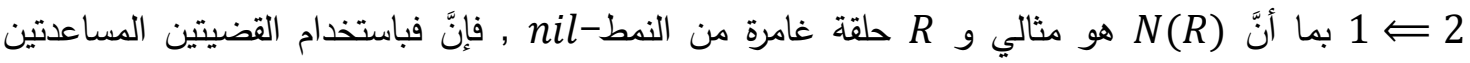
(2.16) و (2.15) نحصل على R حلقة مختزلة. وباستخدام المبرهنة (2.3) نحصل على أن R منتظمة بقوة. 


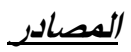

[1] Abdullah , N . K. (2015), " Strongly pure ideals and strongly pure submodules " , K . Un . J./Scientific studies Vol . 10, 12-28 .

[2] AL-Ezeh H.(1988); "The pure spectrum of PF-ring" , comm.Math Univer. S . p . Vol . 37 , No.2, $179-18$.

[3] AL-Ezeh, H.(1989); "pure ideals in commutative reduced Gelf and rings with unity" . Arch . Math . Vol . 53 ,266-269.

[4] Cohn , P - M . (1999), "Reversible rings" , Boll . London Math . Soc ., 31 , 641 -648 .

[5] Mahmood , R.D. (2000) , "On pure ideals and pure sub modules" , Ph . D., Thesis, Mosul university .

[6] Mahmood , R.D. and Mahmood , A.B.(2007),"On rings whose maximal essential ideals are pure" Raf.J.of Comp.and Math.Vol.4,No.1,57-62

[7] Mahmood,R.D.and Mahmood,A.B.(2008),"MaximaL Generalization Of Pure Ideals" Raf .J.of Comp.and Math .Vol . 5 ,No.1,21-27. "

[8] Wei , J . C . and Chen , J . H. (2007); "Nil-injective rings" , Int . Electron . J - of Algebra, Vol .2 1-21.

[9] Wei , J.C, (2013), "Almost abelian rings" , Commun . in Math ., VOL - 21, NO $.1,15-30$.

[10] Zhow , H . (2007), "Left SF-rings and regular rings" Comm . In Algebra, Vol . 35 , NO . $12,3842-3850$. 